\title{
Kommunikation kann man lernen
}

— "Als Erstes heile mit dem Wort" lautet der Untertitel dieses Buches, dementsprechend kommt es auf den ersten Blick wie ein weiterer Kommunikations-Ratgeber daher. In diesen Büchern oder auch in Seminaren zu diesem Thema wird vermittelt, wie man beim Erledigen seiner Aufgaben besser kommunizieren kann. Pamela Emmerling als gelernte Psychotherapeutin und freiberufliche Dozentin setzt den Hebel aber deutlich früher und grundsätzlicher an. Ausgangspunkt war der große Bedarf an gelingender Informationsübermittlung, Konfliktbewältigung und Teamherausbildung. Viele erleben in ihrem Alltag ein Defizit an wirksamen Strategien bei der Kommunikation.

Das Buch bietet zahlreiche Tests an, um die eigene Gesprächsführung zu erkennen und individuelle Stärken und Schwächen zu analysieren. So gerüstet verläuft der Dialog mit Patienten oder Mitarbeitern viel effizienter und nachhaltiger. Mithilfe zahlreicher
Praxisbeispiele gelingt es dann auch, alltägliche Fettnäpfchen und Fallen bei der Kommunikation zu vermeiden. Emmerling vermittelt das hierfür erforderliche Knowhow, um auch schwierige Situationen, in denen wir oft unreflektiert und damit unbefriedigend agieren, passabel meistern können.

Die Autorin hat mit diesem 263-Seiten-Werk ein recht kompaktes Buch geschrieben, das es inhaltlich in sich hat. Der Text ist übersichtlich gestaltet, einige Illustrationen lockern das Layout auf, viele Tabellen, Praxistipps und Schlussfolgerungen (mithilfe von "Dr. No" und „Dr. Will“) unterbrechen gezielt die sonst konsequente Textgestaltung. Sie beschreibt mit viel Humor, treffend und unterhaltsam die täglichen Herausforderungen, berichtet von klassischen Fehlern wie auch von ihren Erfahrungen in verschiedenen Konstellationen - das alles liest sich erfrischend lebensnah. Der Inhalt wird so in einer gelungenen Darstellung "transpor- tiert". Auch die klugen Zusammenfassungen werden nie langweilig.

Angemessen zu kommunizieren, ist im Berufsalltag des Arzt eines der wichtigsten Instrumente. Gespräche sind nicht nur zur Aufklärung und Information da, sie tragen auch zur Heilung bei und sind Teil einer gelungenen Arzt-Patienten-Beziehung. In der Summe zeigt das Buch, dass man Kommunizieren lernen kann, die anschaulich vorgestellten Denk- und Handlungsansätze können in der Praxis eine wertvolle Unterstützung sein. Es ist einerseits ein echtes Arbeitsbuch, andererseits ein anregendes Werk, das viele Denkanstöße und gute Ansätze vermittelt.

Dr. Ulrich Mutschler

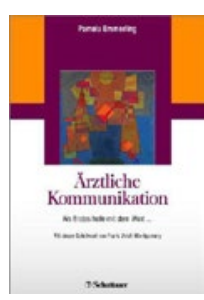

Pamela Emmerling

Ärztliche

Kommunikation

Schattauer-Verlag,

Stuttgart 2014

ISBN 978-3-7945-2974-2

$29,99 €$

\section{Plädoyer eines Kinderarztes}

— Schon an dem Titel kommt kein Kinderarzt vorbei. Und der Untertitel „Ein Kinderarzt empört sich" ist vielen von uns aus der Seele gesprochen. Auf 304 Seiten schreibt sich der Autor seinen Frust, aber auch seine Sorgen vom Herzen: Immer mehr fühlt er sich als "Verordnungsverschreiber" missbraucht. Mütter, mit aus seiner Sicht gesunden Kindern, wenden sich an ihn mit der Bitte um eine Verordnung für Krankengymnastik, Logopädie oder Ergotherapie. Den Termin bei dem Therapeuten haben die besorgten Mütter bereits vereinbart.

Erzieher oder Lehrer üben einen Druck auf die Mütter aus. Schon in der Krabbelgruppe wird erbarmungslos verglichen, den "late talkern" und "late walkern" keine Chance gelassen, sich ihrem Tempo entsprechend zu entwickeln. Die Therapie muss her, da sonst für die Zukunft eine Katastrophe droht.

Differenziert und mit vielen Beispielen aus der Praxis beschreibt der Autor die Zunahme des "Therapiewahns", die Zunahme an Diagnosen bei Kindern, bei denen es sich oft nur um Entwicklungsverzögerungen oder Charaktereigenschaften handelt. Und er schafft es, dass der Leser sich mit ihm empört und sorgt, wo dies alles noch hinführen wird. Vereinzelt schießt der Autor über das Ziel hinaus: So warnt er die Eltern in drastischer Weise vor den bleibenden Folgen von Diagnosen, die die Zukunft des Kindes zerstören können.

Hauch unterstellt seinen Kollegen, in der Diagnosestellung kreativ zu sein, um bessere Abrechnungsmöglichkeiten zu erhalten. Dies mag bei einzelnen der Fall sein, schwarze Schafe gibt es überall. Es jedoch generell zu behaupten, halte ich für grenzwertig. Auch seine Einstellung zur ADHS ist befremdlich: So zitiert er den Arztreport der Barmer GEK als Beleg für eine Inflation in der Diagnosestellung ADHS. Sein kritisches Denken, das er vorher bei Teststatistiken zeigte, verlässt ihn leider dabei. Dass in der KIGGS-Verlaufsstudie keine wesentliche Zunahme der ADHS-Diagnosen seit 2002 beobachtet wurde und vor allem keine Überdiagnose und Übertherapie, wird lieber nicht erwähnt. Bedauerlich finde ich auch die Überzeugung des Autors, dass ADHS nur von Kinder- und Jugendpsychiatern oder in SPZ diagnostiziert und wohl auch therapiert werden solle. Wo bleiben dabei die niedergelassenen Kinder- und Jugendärzte? Seit Jahrzehnten werden Kinder mit ADHS zeitnah, flächendeckend, sorgfältig und kompetent von diesen betreut - zu Zeiten schon als die Diagnose den meisten Kinder- und Jugendpsychiatern inklusive SPZ-Ärzten noch wenig vertraut war. Die AG-ADHS der Kinder- und Jugendärzte mit über 600 Mitgliedern bundesweit sieht es als eine ihrer Aufgaben an, fachliche Kompetenz und Motivation trotz miserabler Honorierung zu erhalten. Ihnen die Kompetenz abzusprechen, in einem Buch, das sich überwiegend an die Eltern von „auffälligen“ Kindern wendet, ist daher für mich nicht nachvollziehbar. Insgesamt ist es dem Autor mit dem Buch „Kindheit ist keine Krankheit" gelungen, ein

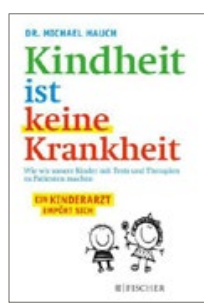

Michael Hauch

\section{Kindheit ist keine} Krankheit

Fischer Taschenbuch Verlag, Frankfurt/Main 2015 ISBN 978-3-596-03230-3 $14,99 €$ 